Proyecciones Journal of Mathematics

Vol. 32, No 1, pp. 31-46, March 2013.

Universidad Católica del Norte

Antofagasta - Chile

\title{
Comparison theorems on fractional order difference equations
}

\author{
J. Jagan Mohan \\ VIT University, India \\ G.V.S.R. Deekshitulu \\ JNTU Kakinada, India \\ Received : November 2011. Accepted : December 2012
}

\begin{abstract}
One of the most efficient methods of obtaining information on the behaviour of solutions of difference equations, even when they cannot be solved explicitly, is the comparison principle. In general, the comparison principle is concerned with estimating a function satisfying a difference inequality by the solution of the corresponding difference equation. In the present paper, we shall establish various forms of the principle for fractional order difference equations.
\end{abstract}

Key Words : Difference equation, under function, over function, fractional order

AMS Classification : 39A10, 39A99. 


\section{Introduction}

Fractional calculus has gained importance during the past three decades due to its applicability in diverse fields of science and engineering. The notions of fractional calculus may be traced back to the works of Euler, but the idea of fractional difference is very recent.

J. B. Diaz and T. J. Osler [8] defined the fractional order difference by the rather natural approach of allowing the index of differencing, in the standard expression for the $n^{\text {th }}$ difference, to be any real or complex number. Later, R.Hirota [9], defined the fractional order difference operator $\nabla^{\alpha}$ where $\alpha$ is any real number, using Taylor's series. Atsushi Nagai [10] adopted another fractional order difference operator by modifying Hirota's [9] operator. Recently, G.V.S.R.Deekshitulu and J.Jagan Mohan [2] modified the definition of Atsushi Nagai [10] for $0<\alpha \leq 1$ in such a way that the expression for $\nabla^{\alpha}$ does not involve any difference operator.

The comparison principles and the nonlinear finite difference inequalities which provide explicit bounds on the unknown functions are the most important and effective tools in the study of nonlinear finite difference equations. In the past few years many new comparison theorems and nonlinear finite difference inequalities have been investigated in order to study the behaviour of solutions of such equations.

In a paper published in 1973, Pachpatte [11] proved the following finite difference inequality which provides the key to the proof of the many basic comparison theorems on finite difference equations.

Theorem 1.1. Let $f(n, r)$ be any function defined for $n \in \mathbf{N}_{0}^{+}, 0 \leq r<\infty$, and nondecreasing with respect to $r$ for any fixed $n \in \mathbf{N}_{0}^{+}$. Let $v(n)$ and $w(n)$ be two functions defined on $\in \mathbf{N}_{0}^{+}$and $u(0) \leq v(0)$.

Assume further that

$$
\begin{aligned}
\nabla v(n+1) & \leq f(n, v(n)) \\
\nabla w(n+1) & \geq f(n, w(n))
\end{aligned}
$$

for all $n \in \mathbf{N}_{0}^{+}$. Then

$$
v(n) \leq w(n)
$$

for all $n \in \mathbf{N}_{0}^{+}$.

In the present paper we present various comparison theorems on various classes of nonlinear fractional order difference equations. 


\section{Preliminaries}

In this section, we introduce some basic definitions and results concerning nabla discrete fractional calculus. Throughout the article, for notations and terminology we refer [1].

Definition 2.1. The extended binomial coefficient $\left(\begin{array}{l}a \\ n\end{array}\right),(a \in \mathbf{R}, n \in \mathbf{Z})$ is defined by

$$
\left(\begin{array}{l}
a \\
n
\end{array}\right)= \begin{cases}\frac{\Gamma(a+1)}{\Gamma(a-n+1) \Gamma(n+1)} & n>0 \\
1 & n=0 \\
0 & n<0 .\end{cases}
$$

In 2003, Atsushi Nagai [10] gave the following definition for fractional order difference operator.

Definition 2.2. Let $\alpha \in \mathbf{R}$ and $m$ be an integer such that $m-1<\alpha \leq m$. The difference operator $\nabla$ of order $\alpha$, with step length $\varepsilon$, is defined as

$\nabla^{\alpha} u(n)= \begin{cases}\nabla^{\alpha-m}\left[\nabla^{m} u(n)\right]=\varepsilon^{m-\alpha} \sum_{j=0}^{n-1}\left(\begin{array}{c}\alpha-m \\ j\end{array}\right)(-1)^{j} \nabla^{m} u(n-j) & \alpha>0 \\ u(n) & \alpha=0 \\ \varepsilon^{-\alpha} \sum_{j=0}^{n-1}\left(\begin{array}{l}\alpha \\ j\end{array}\right)(-1)^{j} u(n-j) & \alpha<0 .\end{cases}$

The above definition contains $\nabla$ operator and the term $(-1)^{j}$ inside the summation index and hence it becomes difficult to study the properties of solutions of fractional difference equations. To avoid this, G.V.S.R.Deekshitulu and J.Jagan Mohan [2] rearranged the terms in Atsushi Nagai's [10] definition as follows, for $\varepsilon=m=1$.

Definition 2.3. The fractional sum operator of order $\alpha(\alpha \in \mathbf{R})$ is defined as

$$
\nabla^{-\alpha} u(n)=\sum_{j=0}^{n-1}\left(\begin{array}{c}
j+\alpha-1 \\
j
\end{array}\right) u(n-j)=\sum_{j=1}^{n}\left(\begin{array}{c}
n-j+\alpha-1 \\
n-j
\end{array}\right) u(j)
$$

and the fractional order difference operator of order $\alpha(\alpha \in \mathbf{R}$ and $0<\alpha \leq$ 1) is defined as

$$
\begin{aligned}
\nabla^{\alpha} u(n) & =\sum_{j=0}^{n-1}\left(\begin{array}{c}
j-\alpha \\
j
\end{array}\right) \nabla u(n-j) \\
& =\sum_{j=1}^{n}\left(\begin{array}{c}
n-j-\alpha-1 \\
n-j
\end{array}\right) u(j)-\left(\begin{array}{c}
n-\alpha-1 \\
n-1
\end{array}\right) u(0) .
\end{aligned}
$$


Theorem 2.1. Let $u(n)$ and $v(n): \mathbf{N}_{0}^{+} \rightarrow \mathbf{R} ; \alpha, \beta \in \mathbf{R}$ such that $0<$ $\alpha, \beta, \alpha+\beta \leq 1$ and $c, d$ are scalars. Then

1. $\nabla^{\beta} \nabla^{\gamma} u(n)=\nabla^{\beta+\gamma} u(n)$.

2. $\nabla^{\alpha}[c u(n)+d v(n)]=c \nabla^{\alpha} u(n)+d \nabla^{\alpha} v(n)$.

3. $\nabla^{-\alpha} \nabla^{\alpha} u(n)=u(n)-u(0)$.

4. $\nabla^{\alpha} \nabla^{-\alpha} u(n)=u(n)$.

5. $\nabla^{\alpha} u(0)=0$ and $\nabla^{\alpha} u(1)=u(1)-u(0)=\nabla u(1)$.

In the following sections we present some basic comparison theorems which can be used as powerful tools in the study of various classes of fractional order difference equations.

\section{Fractional Order Difference Equations}

Definition 3.1. Let $f(n, r)$ be any function defined for $n \in \mathbf{N}_{0}^{+}, 0 \leq r<$ $\infty$ and consider a nonlinear fractional order difference equation of order $\alpha$, $0<\alpha \leq 1$ together with an initial condition as

$$
\nabla^{\alpha} u(n+1)=f(n, u(n)), u(0)=u_{0} .
$$

A function $v(n)$ defined on $\mathbf{N}_{0}^{+}$is said to be an under function with respect to the initial value problem (3.1) if

$$
\nabla^{\alpha} v(n+1) \leq f(n, v(n)) .
$$

Similarly any function $w(n)$ defined on $\mathbf{N}_{0}^{+}$is said to be a over function with respect to the initial value problem (3.1) if

$$
\nabla^{\alpha} w(n+1) \geq f(n, w(n)) .
$$

The present section deals with a nonlinear fractional order difference equation of the form (3.1) where $f(n, r)$ is a nonnegative and nondecreasing function with respect to $r$ for any fixed $n \in \mathbf{N}_{0}^{+}$.

Theorem 3.1. [2] Let $v(n)$ and $w(n)$ are under and over functions with respect to the initial value problem (3.1).

Then $v(0) \leq w(0)$ implies

$$
v(n) \leq w(n)
$$

for all $n \in \mathbf{N}_{0}^{+}$. 
Remark 1. We note that the conclusion of Theorem 3.1 remains valid if we replace (3.2) and (3.3) by

$$
\nabla^{\alpha} v(n+1)-f(n, v(n)) \leq \nabla^{\alpha} w(n+1)-f(n, w(n))
$$

If we assume that $u(0)<v(0)$ in Theorem 3.1, the equality in the conclusion (3.4) must be dropped. We also note that the conditions given in (3.2) and (3.3) can also be written in the form

$$
\begin{gathered}
v(n) \leq v(0)+\sum_{j=0}^{n-1}\left(\begin{array}{c}
n-j+\alpha-2 \\
n-j-1
\end{array}\right) f(j, v(j)) \\
w(n) \geq w(0)+\sum_{j=0}^{n-1}\left(\begin{array}{c}
n-j+\alpha-2 \\
n-j-1
\end{array}\right) f(j, w(j))
\end{gathered}
$$

and

$v(n)-v(0)+\sum_{j=0}^{n-1}\left(\begin{array}{c}n-j+\alpha-2 \\ n-j-1\end{array}\right) f(j, v(j)) \leq w(n)-w(0)+\sum_{j=0}^{n-1}\left(\begin{array}{c}n-j+\alpha-2 \\ n-j-1\end{array}\right) f(j, w(j))$

respectively.

Theorem 3.2. [2] Let $v(n)$ and $w(n)$ are under and over functions with respect to the initial value problem (3.1) such that $v(0) \leq u(0) \leq w(0)$. Then

$$
v(n) \leq u(n) \leq w(n)
$$

for all $n \in \mathbf{N}_{0}^{+}$.

Remark 2. The relations $v(n)<u(n)$ and $w(n)>u(n)$ remain valid for $n \in \mathbf{N}_{0}^{+}$, when the inequalities (3.2) and (3.3) are replaced by

$$
\begin{aligned}
\nabla^{\alpha} v(n+1) & <f(n, v(n)), \\
\nabla^{\alpha} w(n+1) & >f(n, w(n))
\end{aligned}
$$

for $n \in \mathbf{N}_{0}^{+}$, provided the solution of (3.1) exists for all $n \in \mathbf{N}_{0}^{+}$.

Theorem 3.3. Let $g(n, r)$ be any function defined for $n \in \mathbf{N}_{0}^{+}, 0 \leq r<\infty$. Then, if an inequality

$$
|g(n, r)| \leq f(n,|r|)
$$


is satisfied, there holds an inequality

$$
|x(n)| \leq y(n),
$$

$n \in \mathbf{N}_{0}^{+}$provided $\left|x_{0}\right| \leq y_{0}$, where $x(n)$ and $y(n)$ are the solutions

$$
\begin{aligned}
& \nabla^{\alpha} x(n+1)=g(n, x(n)), x(0)=x_{0}, \\
& \nabla^{\alpha} y(n+1)=f(n, y(n)), y(0)=y_{0},
\end{aligned}
$$

respectively.

Proof. Suppose that (3.13) is not true. Then there exists a $k \in \mathbf{N}_{0}^{+}$ such that $|x(j)| \leq y(j)$ for $j \leq k$ and

$$
|x(k+1)|>y(k+1) .
$$

Now using the monotone property of $f$, for $j \leq k$ and (2.3),

$$
\begin{aligned}
|x(k+1)| & =\left|\left(\begin{array}{c}
k-\alpha \\
k
\end{array}\right) x(0)+\alpha \sum_{j=1}^{k} \frac{1}{(j-\alpha)}\left(\begin{array}{c}
j-\alpha \\
j
\end{array}\right) x(k+1-j)+g(j, x(j))\right| \\
& \leq\left(\begin{array}{c}
k-\alpha \\
k
\end{array}\right)|x(0)|+\alpha \sum_{j=1}^{k} \frac{1}{(j-\alpha)}\left(\begin{array}{c}
j-\alpha \\
j
\end{array}\right)|x(k+1-j)|+|g(j, x(j))| \\
& \leq\left(\begin{array}{c}
k-\alpha \\
k
\end{array}\right) y(0)+\alpha \sum_{j=1}^{k} \frac{1}{(j-\alpha)}\left(\begin{array}{c}
j-\alpha \\
j
\end{array}\right) y(k+1-j)+f(j,|x(j)|) \\
& \leq\left(\begin{array}{c}
k-\alpha \\
k
\end{array}\right) y(0)+\alpha \sum_{j=1}^{k} \frac{1}{(j-\alpha)}\left(\begin{array}{c}
j-\alpha \\
j
\end{array}\right) y(k+1-j)+f(j, y(j))
\end{aligned}
$$

which is a contradiction to (3.16). Hence the proof.

Theorem 3.4. [2] Let $m_{1}(n, r)$ and $m_{2}(n, r)$ be two nonnegative functions defined for $n \in \mathbf{N}_{0}^{+}, 0 \leq r<\infty$ and nondecreasing with respect to $r$ for any fixed $n \in \mathbf{N}_{0}^{+}$. Let $r(n)$ be a function defined for $n \in \mathbf{N}_{0}^{+}$and that

$$
m_{1}(n, r(n)) \leq \nabla^{\alpha} r(n+1) \leq m_{2}(n, r(n))
$$

for all $n \in \mathbf{N}_{0}^{+}$. Let $v(n)$ and $w(n)$ be the solutions of the difference equations

$$
\begin{aligned}
& \nabla^{\alpha} x(n+1)=m_{1}(n, x(n)), x(0)=x_{0} \\
& \nabla^{\alpha} y(n+1)=m_{2}(n, y(n)), y(0)=y_{0}
\end{aligned}
$$


and suppose that $x_{0} \leq r(0) \leq y_{0}$. Then

$$
x(n) \leq r(n) \leq y(n), n \in \mathbf{N}_{0}^{+} .
$$

Theorem 3.5. [2] Let $h(n, r, s)$ be a function defined for $n \in \mathbf{N}_{0}^{+}, 0 \leq r<$ $\infty, 0 \leq s<\infty$ is nonnegative and nondecreasing with respect to $r$ and $s$ for any fixed $n \in \mathbf{N}_{0}^{+}$. Let $r(n)$ be solution of the difference equation

$$
\nabla^{\alpha} r(n+1)=h(n, r(n), r(n)), r(0)=r_{0}
$$

for all $n \in \mathbf{N}_{0}^{+}$. Suppose that the inequality

$$
\nabla^{\alpha} x(n+1) \leq h(n, x(n), y(n))
$$

is satisfied for all $n \in \mathbf{N}_{0}^{+}$, where the functions $x(n)$ and $y(n)$ are defined for $n \in \mathbf{N}_{0}^{+}$such that $x(0) \leq r_{0}$. Then

$$
x(n) \leq r(n)
$$

for all $n \in \mathbf{N}_{0}^{+}$provided

$$
y(n) \leq r(n)
$$

for all $n \in \mathbf{N}_{0}^{+}$.

Theorem 3.6. Let $y(n), a(n)$ and $b(n)$ be nonnegative functions defined for $n \in \mathbf{N}_{0}^{+}$. If

$$
y(n) \leq a(n)+b(n) \sum_{j=0}^{n-1}\left(\begin{array}{c}
n-j+\alpha-2 \\
n-j-1
\end{array}\right) u(j)
$$

for $n \in \mathbf{N}_{0}^{+}$, then

$$
y(n) \leq a(n)+b(n) r(n)
$$

for $n \in \mathbf{N}_{0}^{+}$, where $r(n)$ is the solution of the fractional order difference equation

$$
\nabla^{\alpha} r(n+1)=f(n, a(n)+b(n) r(n)), r(0)=0
$$

for $n \in \mathbf{N}_{0}^{+}$. 
Proof. Define function $z(n)$ by

$$
z(n)=\sum_{j=0}^{n-1}\left(\begin{array}{c}
n-j+\alpha-2 \\
n-j-1
\end{array}\right) u(j) .
$$

Then $z(0)=0, y(n) \leq a(n)+b(n) z(n)$ and

$$
\nabla^{\alpha} z(n+1)=f(n, y(n)) \leq f(n, a(n)+b(n) z(n)) .
$$

By using Theorem 3.4, we have $z(n) \leq r(n)$. Then $y(n) \leq a(n)+$ $b(n) z(n) \leq a(n)+b(n) r(n)$. Hence the proof.

\section{Fractional Order Difference Equations of Volterra Type}

Definition 4.1. Let $f(n, r, s)$ be a function defined for $n \in \mathbf{N}_{0}^{+}, 0 \leq r<$ $\infty, 0 \leq s<\infty$ and $g(n, m, r)$ be a function defined for $n, m \in \mathbf{N}_{0}^{+}, m \leq n$, $0 \leq r<\infty$. Let $u(n)$ be a function defined for $n \in \mathbf{N}_{0}^{+}$.

Then a nonlinear fractional difference equation of Volterra type of order $\alpha, 0<\alpha \leq 1$ is of the form

$$
\nabla^{\alpha} u(n+1)=f\left(n, u(n), \sum_{m=0}^{n-1} g(n, m, u(m))\right), u(0)=u_{0} .
$$

A function $v(n)$ defined on $\mathbf{N}_{0}^{+}$is said to be an under function with respect to the initial value problem (4.1) if

$$
\nabla^{\alpha} v(n+1) \leq f\left(n, v(n), \sum_{m=0}^{n-1} g(n, m, v(m))\right) .
$$

Similarly any function $w(n)$ defined on $\mathbf{N}_{0}^{+}$is said to be a over function with respect to the initial value problem (4.1) if

$$
\nabla^{\alpha} w(n+1) \geq f\left(n, w(n), \sum_{m=0}^{n-1} g(n, m, w(m))\right) .
$$

In this section we deal with a nonlinear fractional difference equation of Volterra type of the form (4.1) where $f(n, r, s)$ is a nonnegative and nondecreasing function with respect to $r$ and $s, 0 \leq r, s<\infty$ for any fixed $n \in \mathbf{N}_{0}^{+}$and $g(n, m, r)$ is a nonnegative and nondecreasing function with respect to $r, 0 \leq r<\infty$ for any fixed $n, m \in \mathbf{N}_{0}^{+}$. 
Theorem 4.1. [4] Let $v(n)$ and $w(n)$ are lower and under functions with respect to the initial value problem (4.1). Then $v(0) \leq w(0)$ implies

$$
v(n) \leq w(n)
$$

for all $n \in \mathbf{N}_{0}^{+}$.

Theorem 4.2. [4] Let $h(n, r)$ be a nonnegative function defined for $n \in$ $\mathbf{N}_{0}^{+}, 0 \leq r<\infty$. Let $x(n)$ and $y(n)$ be any two nonnegative functions defined for $n \in \mathbf{N}_{0}^{+}$. Suppose that for $n \in \mathbf{N}_{0}^{+}$, the inequalities

$$
\begin{aligned}
& \nabla^{\alpha} x(n+1) \leq h(n, x(n))+\sum_{m=0}^{n-1} g(n, m, x(m)), \\
& \nabla^{\alpha} y(n+1) \geq h(n, y(n))+\sum_{m=0}^{n-1} g(n, m, y(m))
\end{aligned}
$$

hold. If for any $n \in \mathbf{N}_{0}^{+}$,

$$
h(n, x(n))-h(n, y(n)) \leq-\alpha(x(n)-y(n))
$$

then $x(0) \leq y(0)$ implies

$$
x(n) \leq y(n)
$$

for all $n \in \mathbf{N}_{0}^{+}$.

Theorem 4.3. [4] Let $v(n)$ and $w(n)$ are lower and under functions with respect to the initial value problem (4.1) such that $v(0) \leq u(0) \leq w(0)$. Then

$$
v(n) \leq u(n) \leq w(n)
$$

respectively for all $n \in \mathbf{N}_{0}^{+}$.

Theorem 4.4. [4] Let $f_{1}(n, r, s)$ and $f_{2}(n, r, s)$ be two nonnegative and nondecreasing function with respect to $r$ and $s, 0 \leq r, s<\infty$ for any fixed $n \in \mathbf{N}_{0}^{+}$and $g_{1}(n, m, r)$ and $g_{2}(n, m, r)$ be a nonnegative and nondecreasing function with respect to $r, 0 \leq r<\infty$ for any fixed $n, m \in \mathbf{N}_{0}^{+}$. Let $r(n)$ be a function defined for $n \in \mathbf{N}_{0}^{+}$and that

$f_{1}\left(n, r(n), \sum_{m=0}^{n-1} g_{1}(n, m, r(m))\right) \leq \nabla^{\alpha} r(n+1) \leq f_{2}\left(n, r(n), \sum_{m=0}^{n-1} g_{2}(n, m, r(m))\right)$ 
for all $n \in \mathbf{N}_{0}^{+}$. Let $x(n)$ and $y(n)$ be the solutions of the difference equations

$$
\begin{aligned}
& \nabla^{\alpha} x(n+1)=f_{1}\left(n, x(n), \sum_{m=0}^{n-1} g_{1}(n, m, x(m))\right), x(0)=x_{0} \\
& \nabla^{\alpha} y(n+1)=f_{2}\left(n, y(n), \sum_{m=0}^{n-1} g_{2}(n, m, y(m))\right), y(0)=y_{0}
\end{aligned}
$$

and suppose that $x_{0} \leq r(0) \leq y_{0}$. Then

$$
x(n) \leq r(n) \leq w(n), n \in \mathbf{N}_{0}^{+} .
$$

Theorem 4.5. [4] Let $x(n)$ and $a(n)$ be two nonnegative functions defined for $n \in \mathbf{N}_{0}^{+}$. Let $h(n, m)$ be a nonnegative function defined for $n, m \in N_{0}^{+}$. If the inequality

$(4.14) \nabla^{\alpha} x(n+1) \leq a(n)+\sum_{m=0}^{n-1} h(n, m) f\left(m, x(m), \sum_{j=0}^{m-1} g(m, j, x(j))\right)$

is satisfied for all $n \in \mathbf{N}_{0}^{+}$. Then $x(0) \leq r(0)$ implies

$$
x(n) \leq r(n)
$$

where $r(n)$ is solution of the difference equation

$\nabla^{\alpha} r(n+1)=a(n)+\sum_{m=0}^{n-1} h(n, m) f\left(m, r(m), \sum_{j=0}^{m-1} g(m, j, r(j))\right), r(0)=a(0)$

for all $n \in \mathbf{N}_{0}^{+}$.

Corollary 4.5.1. Let $x(n), y(n)$ and $a(n)$ be any nonnegative functions defined for $n \in \mathbf{N}_{0}^{+}$. Let $h(n, m)$ be a nonnegative function defined for $n$, $m \in \mathbf{N}_{0}^{+}$. If the inequality

$$
\nabla^{\alpha} x(n+1) \leq a(n)+\sum_{m=0}^{n-1} h(n, m) f(m, x(m), y(m))
$$

is satisfied for all $n \in \mathbf{N}_{0}^{+}$. Then $x(0) \leq r(0)$ implies

$$
x(n) \leq r(n)
$$


provided

$$
y(n) \leq r(n)
$$

where $r(n)$ is solution of the fractional order difference equation

$(4.20) \nabla^{\alpha} r(n+1)=a(n)+\sum_{m=0}^{n-1} h(n, m) f(m, r(m), y(m)), r(0)=a(0)$

for all $n \in \mathbf{N}_{0}^{+}$.

Theorem 4.6. Let $y(n)$ and $a(n)$ be nonnegative functions defined for $n \in \mathbf{N}_{0}^{+}$. If

$$
\nabla^{\alpha} y(n+1) \leq a(n)+\sum_{j=0}^{n-1} g(n, j, u(j))
$$

for $n \in \mathbf{N}_{0}^{+}$, then

$$
u(n) \leq r(n)
$$

for $n \in \mathbf{N}_{0}^{+}$, where $r(n)$ is the solution of the fractional order difference equation

$$
\nabla^{\alpha} r(n+1)=a(n)+\sum_{j=0}^{n-1} g(n, j, r(j))
$$

for $n \in \mathbf{N}_{0}^{+}$.

Proof. Suppose that (4.22) is not true. Then there exists a $k \in \mathbf{N}_{0}^{+}$ such that $y(j) \leq r(j)$ for $j \leq k$ and

$$
y(k+1)>r(k+1) .
$$

From the monotone property of $f$, for $j \leq k$,

$$
\begin{aligned}
y(j) \leq r(j) & \Rightarrow g(k, j, y(j)) \leq g(k, j, r(j)) \\
& \Rightarrow \sum_{m=0}^{k-1} g(k, j, y(j)) \leq \sum_{j=0}^{k-1} g(k, j, r(j)) .
\end{aligned}
$$

Now using (2.3) and (4.25),

$$
\begin{aligned}
y(k+1) & \leq\left(\begin{array}{c}
k-\alpha \\
k
\end{array}\right) y(0)+\alpha \sum_{j=1}^{k} \frac{1}{(j-\alpha)}\left(\begin{array}{c}
j-\alpha \\
j
\end{array}\right) y(k+1-j)+\sum_{j=0}^{k-1} g(k, j, y(j)) \\
& \leq\left(\begin{array}{c}
k-\alpha \\
k
\end{array}\right) r(0)+\alpha \sum_{j=1}^{k} \frac{1}{(j-\alpha)}\left(\begin{array}{c}
j-\alpha \\
j
\end{array}\right) r(k+1-j)+\sum_{j=0}^{k-1} g(k, j, r(j)) \\
& =r(k+1)
\end{aligned}
$$


which is a contradiction to (4.24). Hence the proof.

Remark 3. If the inequality given in (4.21) is replaced by

$$
\nabla^{\alpha} y(n+1) \geq a(n)+\sum_{j=0}^{n-1} g(n, j, y(j))
$$

then the conclusion (4.22) in Theorem 4.6 reduces to $y(n) \geq r(n)$ for $n \in$ $\mathbf{N}_{0}^{+}$.

Theorem 4.7. Let $x(n)$ and $y(n)$ be solutions of the fractional order difference equations

$$
\begin{aligned}
& \nabla^{\alpha} x(n+1)=a_{1}(n)+\sum_{j=0}^{n-1} g_{1}(n, j, x(j)) \\
& \nabla^{\alpha} y(n+1)=a_{2}(n)+\sum_{j=0}^{n-1} g_{2}(n, j, y(j))
\end{aligned}
$$

where the functions $x(n), y(n), a_{1}(n), a_{2}(n), g_{1}(n, m, r)$ and $g_{2}(n, m, s)$ are defined for $n, m \in \mathbf{N}_{0}^{+}, m \leq n, 0 \leq r, s<\infty$ and satisfy the conditions

$$
\left|g_{1}(n, m, r)-g_{2}(n, m, s)\right| \leq g(n, m,|r-s|)
$$

for all $n, m \in \mathbf{N}_{0}^{+}, m \leq n, 0 \leq r, s<\infty$. Let $r(n)$ be any solution of the fractional order difference equation

$$
\nabla^{\alpha} r(n+1)=a(n)+\sum_{j=0}^{n-1} g(n, j, r(j))
$$

for $n \in \mathbf{N}_{0}^{+}$. If

$$
\left|a_{1}(n)-a_{2}(n)\right| \leq a(n)
$$

then

$$
|x(n)-y(n)| \leq r(n)
$$

for all $n \in \mathbf{N}_{0}^{+}$. 
Proof. Define a function $z(n)$ by $z(n)=|x(n)-y(n)|$. Suppose that (4.32) is not true. Then there exists a $k \in \mathbf{N}_{0}^{+}$such that $z(j) \leq r(j)$ for $j \leq k$ and

$$
z(k+1)>r(k+1)
$$

Consider

$$
\begin{aligned}
z(k+1) & =\mid\left(\begin{array}{c}
k-\alpha \\
k
\end{array}\right) x(0)+\alpha \sum_{j=1}^{k} \frac{1}{(j-\alpha)}\left(\begin{array}{c}
j-\alpha \\
j
\end{array}\right) x(k+1-j)+a_{1}(k)+\sum_{j=0}^{k} g_{1}(k, j, x(j)) \\
& -\left(\begin{array}{c}
k-\alpha \\
k
\end{array}\right) y(0)-\alpha \sum_{j=1}^{k} \frac{1}{(j-\alpha)}\left(\begin{array}{c}
j-\alpha \\
j
\end{array}\right) y(k+1-j)+a_{2}(k)+\sum_{j=0}^{k} g_{2}(k, j, y(j)) \mid \\
& \leq\left(\begin{array}{c}
k-\alpha \\
k
\end{array}\right)|x(0)-y(0)|+\alpha \sum_{j=1}^{k} \frac{1}{(j-\alpha)}\left(\begin{array}{c}
j-\alpha \\
j
\end{array}\right)|x(k+1-j)-y(k+1-j)| \\
& +\left|a_{1}(k)-a_{2}(k)\right|+\sum_{j=0}^{k}\left|g_{1}(k, j, x(j))-g_{2}(k, j, y(j))\right| \\
& \leq\left(\begin{array}{c}
k-\alpha \\
k
\end{array}\right) z(0)+\alpha \sum_{j=1}^{k} \frac{1}{(j-\alpha)}\left(\begin{array}{c}
j-\alpha \\
j
\end{array}\right) z(k+1-j)+a(k)+\sum_{j=0}^{k} g(k, j, z(j)) \\
& \leq\left(\begin{array}{c}
k-\alpha \\
k
\end{array}\right) r(0)+\alpha \sum_{j=1}^{k} \frac{1}{(j-\alpha)}\left(\begin{array}{c}
j-\alpha \\
j
\end{array}\right) r(k+1-j)+a(k)+\sum_{j=0}^{k} g(k, j, r(j)) \\
& =r(k+1) .
\end{aligned}
$$

which is a contradiction to (4.33). Hence the proof.

Corollary 4.7.1. [4] Let $f$ be a nonnegative and nondecreasing function with respect to its arguments. Let $r(n)$ be solution of the fractional order difference equation

$$
\nabla^{\alpha} r(n+)=f(r(n), r(n-1), \ldots \ldots . . r(n-k))
$$

for all $n \in \mathbf{N}_{0}^{+}$. Suppose that the inequality

$$
\nabla^{\alpha} x(n+1) \leq f(x(n), x(n-1), \ldots \ldots \ldots x(n-k))
$$

is satisfied for all $n \in \mathbf{N}_{0}^{+}$, where $x(j)(j=0,1,2 \ldots)$ is a positive sequence of functions defined for $n \in \mathbf{N}_{0}^{+}$such that $x(j) \leq r(j), j=0,1, \ldots . k$. Then

$$
x(n) \leq r(n)
$$

for all $n \in \mathbf{N}_{0}^{+}$. 
Theorem 4.8. [4] Let $f$ be a nonnegative and nondecreasing function with respect to its arguments. Let $r(n)$ be solution of the difference equation

$$
\nabla^{\alpha} r(n+1)=f\left(r(n), \sum_{j=0}^{n-1} r(j), \sum_{j=0}^{n-2} r(j)\right)
$$

for all $n \in \mathbf{N}_{0}^{+}$. Suppose that the inequality

$$
\nabla^{\alpha} y(n+1)=f\left(y(n), \sum_{j=0}^{n-1} y(j), \sum_{j=0}^{n-2} y(j)\right)
$$

is satisfied for all $n \in \mathbf{N}_{0}^{+}$, where $y(j)(j=0,1,2 \ldots)$ is a positive sequence of functions defined for $n \in \mathbf{N}_{0}^{+}$such that $y(0) \leq r(0)$. Then

$$
y(n) \leq r(n)
$$

for all $n \in \mathbf{N}_{0}^{+}$.

\section{References}

[1] Agarwal, R. P. Difference equations and inequalities, Marcel Dekker, New York, (1992).

[2] Deekshitulu, G. V. S. R. and Jagan Mohan, J. Fractional difference inequalities, Communications in Applied Analysis, 14 (2010), No. 1, pp. 89 - 98.

[3] Deekshitulu, G. V. S. R. and Jagan Mohan, J. Fractional difference inequalities of Bihari type, Communications in Applied Analysis, 14 (2010), No. 4, pp. 343 - 354.

[4] Deekshitulu, G. V. S. R. and Jagan Mohan, J. Fractional difference inequalities of Volterra type, International Journal of Pure and Applied Mathematics, 70 (2011), No. 2, pp. 137 - 149.

[5] Deekshitulu, G. V. S. R. and Jagan Mohan, J. Fractional difference inequalities of Opial type and initial value problem, Fractional Differential Calculus, 2 (2012), No. 1, pp. 73 - 86. 
[6] Deekshitulu, G. V. S. R. and Jagan Mohan, J. Some New Fractional Difference Inequalities, ICMMSC 2012, CCIS 283 (2012), SpringerVerlag, Berlin, Heidelberg, pp. 403 - 412.

[7] Deekshitulu, G. V. S. R. and Jagan Mohan, J. Some New Fractional Difference Inequalities of Gronwall - Bellman Type, Mathematical Sciences, Springer Open, Volume 6, Number 69, doi: 10.1186/2251-74566-69.

[8] Diaz, J. B. and Osler, T. J. Differences of fractional order, Math. Comp., 28, pp. 185-201, (1974).

[9] Hirota, R. Lectures on difference equations, Science-sha, 2000 (in Japanese).

[10] Nagai, A. An integrable mapping with fractional difference, J. Phys. Soc. Jpn. 72, pp. 21812183, (2003).

[11] Pachpatte, B. G. Inequalities of finite difference equations, Marcel Dekker, New York, (2002).

[12] Podlubny, I Fractional differential equations, Academic press, San Diego, (1999).

[13] S. Sugiyama Comparison theorems on difference equations, Bull. Sci. Engr. Research Lab., Waseda Univ., 47, pp. 77-82, (1970).

\author{
J. Jagan Mohan \\ Fluid Dynamics Division, \\ School of Advanced Sciences, \\ VIT University, \\ Vellore - 632014, \\ Tamil Nadu, \\ India \\ e-mail : j.jaganmohan@hotmail.com \\ and
}


G. V. S. R. Deekshitulu

Department of Mathematics,

JNTU Kakinada

Kakinada

Andhra Pradesh - 533003

India

e-mail : dixitgvsr@hotmail.com 